\title{
Ultrafast time-resolved spectroscopy of a fs-laser-induced plasma inside glass using a super-continuum probe beam
}

\author{
Javier Hernandez-Rueda ${ }^{1}$ D . Jonathan J. Witcher ${ }^{1} \cdot$ Denise M. Krol ${ }^{1}$
}

Received: 21 June 2019 / Accepted: 31 July 2019 / Published online: 6 August 2019

(c) The Author(s) 2019

\begin{abstract}
The light-energy coupling during femtosecond laser processing of glass is mediated by non-linear ionization mechanisms through the formation of an electron plasma. Its transient optical properties provide information about the density, temperature and scattering rate of the excited electrons. In turn, these properties strongly condition the features and size of the permanent optical modification near the focal volume that are desirable to fabricate photonic devices, such as optical waveguides inside transparent materials. Here, we report on the spectral response of a fs-laser-induced electron plasma inside fused silica by measuring its transient transmission using a broadband probe. We model the interaction of the probe beam with the plasma by combining Drude-Sommerfeld model with Gaussian optics. In this manner, we take into account both the laser-plasma interaction and the influence of the chromatic aberration inherent to a broadband-based system. We find good agreement between experiments at several processing energies and simulations and provide an estimate of the dielectric function of the excited material.
\end{abstract}

\section{Introduction}

Ultrafast laser ablation and micromachining of transparent materials with ultrashort laser pulses has drawn the attention of an ever-increasing number of research groups over the last decades [1-6]. Direct laser writing is the cornerstone of laser processing in bulk dielectrics, for instance, to inscribe optical waveguides as demonstrated for the first time by Hirao and Miura more than 20 years ago [7-9]. This technique is based on the inscription of 3D structures inside transparent materials by locally modifying the refractive index using a train of tightly focused fs-laser pulses while translating the sample, which offers an unprecedented spatial resolution and has been demonstrated in several crystalline and amorphous dielectrics, i.e., diamond [10], sapphire [11], quartz [11], fused silica [11-17], borosilicate glass [18], phosphotellurite glass [19, 20] and zinc phosphate glasses [21, 22].

Javier Hernandez-Rueda

fjavihr@gmail.com

1 Department of Materials Science and Engineering, University of California Davis, Davis, CA 95616, USA

2 Present Address: Kavli Institute of Nanoscience, Department of Quantum Nanoscience, Delft University of Technology, 2628 CJ Delft, The Netherlands
The interaction mechanisms of fs-laser pulses with dielectrics have been intensely investigated to understand and control the energy deposition mechanisms that, in turn, lead to the desired permanent modification. The laser-matter interaction is mainly mediated by non-linear absorption. During the first hundreds of femtoseconds, a dense and hot free electron plasma is excited by means of multiphoton ionization and avalanche ionization [23, 24]. On the picosecond time scale, the free electrons thermalize with the lattice, inducing a local temperature increase that may lead to a phase change and subsequently to optical changes [25, 26]. Experimental studies based on time-resolved techniques are particularly relevant to elucidate the precise nature of these physical mechanisms. Most of these works are based on time-resolved microscopy arrangements that image the transient optical properties of the laser-excited region, i.e., phase contrast microscopy [26, 27], time-resolved transmission (shadowgraphy) [28-32] and microscopy in reflection mode $[33,34]$. These provide valuable information about the free electron plasma properties, i.e., rise time, density and scattering time of the electrons, and of the transient refractive index of the irradiated region over several time scales. It also yields access to both temporal and spatial features of the process, although limited to a monochromatic probe beam with a relatively narrow spectral bandwidth, typically below $\Delta \lambda<10 \mathrm{~nm}$, that does not provide spectral information. 
We recently reported on the transient transmission spectroscopy of glass during fs-laser writing using a single processing energy [31]. However, we could not accurately explain the precise spectral response of the laser-induced plasma by simply applying the Drude formula nor relate it to the final modification. In this letter, we present the temporally and spectrally resolved transmission of a fs-laserinduced plasma inside fused silica, which is produced using several processing energies that ultimately lead to either waveguides or tracks of damaged material. We combine Gaussian beam optics with the Drude model to compute the frequency-resolved transmission. In particular, we introduce an effective transmission $\mathcal{T}(\omega, \mathbf{r})$ function equal to the scalar product of the electron gas response function and the chromatic aberration inherent to our broadband-based system. We emphasize the implications of using a super-continuum probe on the features of the transient transmission to accurately reproduce the experimental results. Finally, we show the processing energy dependence of the electron plasma properties retrieved using the model and experiments and link those to the imprinted final modification.

\section{Experimental}

Figure 1a shows an example of fs-laser inscribed lines inside fused silica using direct laser writing [16]. The images illustrate the lateral view and the cross-section of a track of damage (top) and a waveguide (below). Figure $1 \mathrm{~b}$ shows a diagram of the pump and probe experimental setup, which is described in greater detail elsewhere [31]. We used the setup to investigate the transient transmission during laser processing for energies that lead to the permanent tracks shown in Fig. 1a. The sample consists of polished $(\approx \lambda / 10)$ standard grade fused silica (7980 Corning). For the experiments, we use a fs-laser amplifier (Spectra-Physics, Spitfire LCX) that generates pulses at $800 \mathrm{~nm}$ with a pulse duration of $\Delta t=200$ fs at full-width half-maximum that was characterized using an autocorrelator (SSA Spectra-Physics). A laser pulse is split into pump and probe using a polarizing beam splitter. The pump passes through an energy control system and is then tightly focused inside the glass sample (depth $\approx 1 \mathrm{~mm}$ ) using a microscope objective (Olympus Plan ELWD 20×, $\mathrm{NA}=0.40$, infinity corrected). The probe pulse runs over a motorized delay line (Aerotech, maximum delay of $1 \mathrm{~ns}$ ) and passes through a sapphire plate $(500 \mu \mathrm{m})$ to produce white light via super-continuum (SC) generation, thus resulting in a broadband SC probe. Afterwards, the probe is steered towards a microscope objective (Nikon CFI Plan 50×, NA $=0.55$, ELWD, infinity corrected) that focuses it such that pump ( $z$-axis) and probe ( $x$-axis) are spatially overlapped at $90^{\circ}$. Note that this objective is designed to correct for lateral and axial chromatic aberrations. The transmitted SC probe light is collected by a lens, filtered (notch filter at 808 $\mathrm{nm}$ ) and sent to the spectrometers (Ocean Optics and Hamamatsu) or to the imaging system (Sony XC77 camera). The inset shows micrographs of the $z-y$ plane to better illustrate the geometry of the system. They show the pump-induced plasma emission and the attenuated SC probe. Note that we account for the plasma emission at each excitation energy by measuring a spectrum while blocking the probe [31]. This curve is then subtracted to the spectra measured with both pump and probe beams present.

\section{Results}

Using the experimental setup, we measure the temporally and spectrally resolved transmission of the fs-laser-excited glass, acquired within a spectral range that spans from 500 to $1100 \mathrm{~nm}$. Figure 2a reproduces the transmission curves at several time delays using the pump laser energy of $4 \mu \mathrm{J}$ used in our previous work [31]. The raw spectra are normalized calculating the ratio of the SC probe signal acquired with and without the pump beam after subtracting the plasma emission. The curve collected at 0 fs presents a slight
Fig. 1 a Microscopy images of a track of damaged material and a waveguide machined inside fused silica. b Experimental setup for transient spectra acquisition through a fs-laser-excited volume inside fused silica. The inset shows micrographs of the pump and the probe in the $z-y$ plane and a cross-section of the pump along the $y$-axis (a)

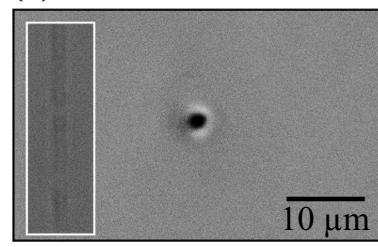

(b)

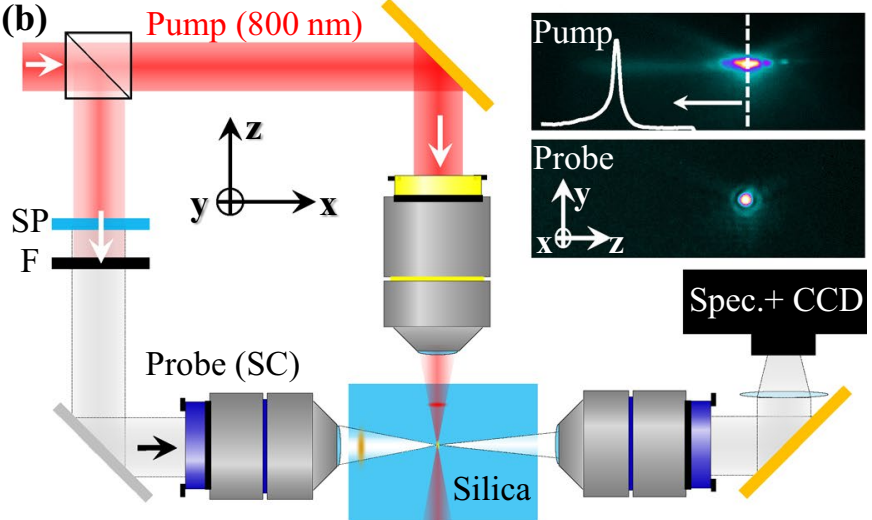


Fig. 2 a Experimental transient transmission of the electron plasma as a function of wavelength at several time delays. The laser pump energy employed during the experiment was $E_{\text {pump }}=4 \mu \mathrm{J}$. b Transient transmission of the electron plasma at 118 ps using three pump pulses of energy $0.7 \mu \mathrm{J}$ (green), $1.3 \mu \mathrm{J}$ (red) and $4.0 \mu \mathrm{J}$ (blue)
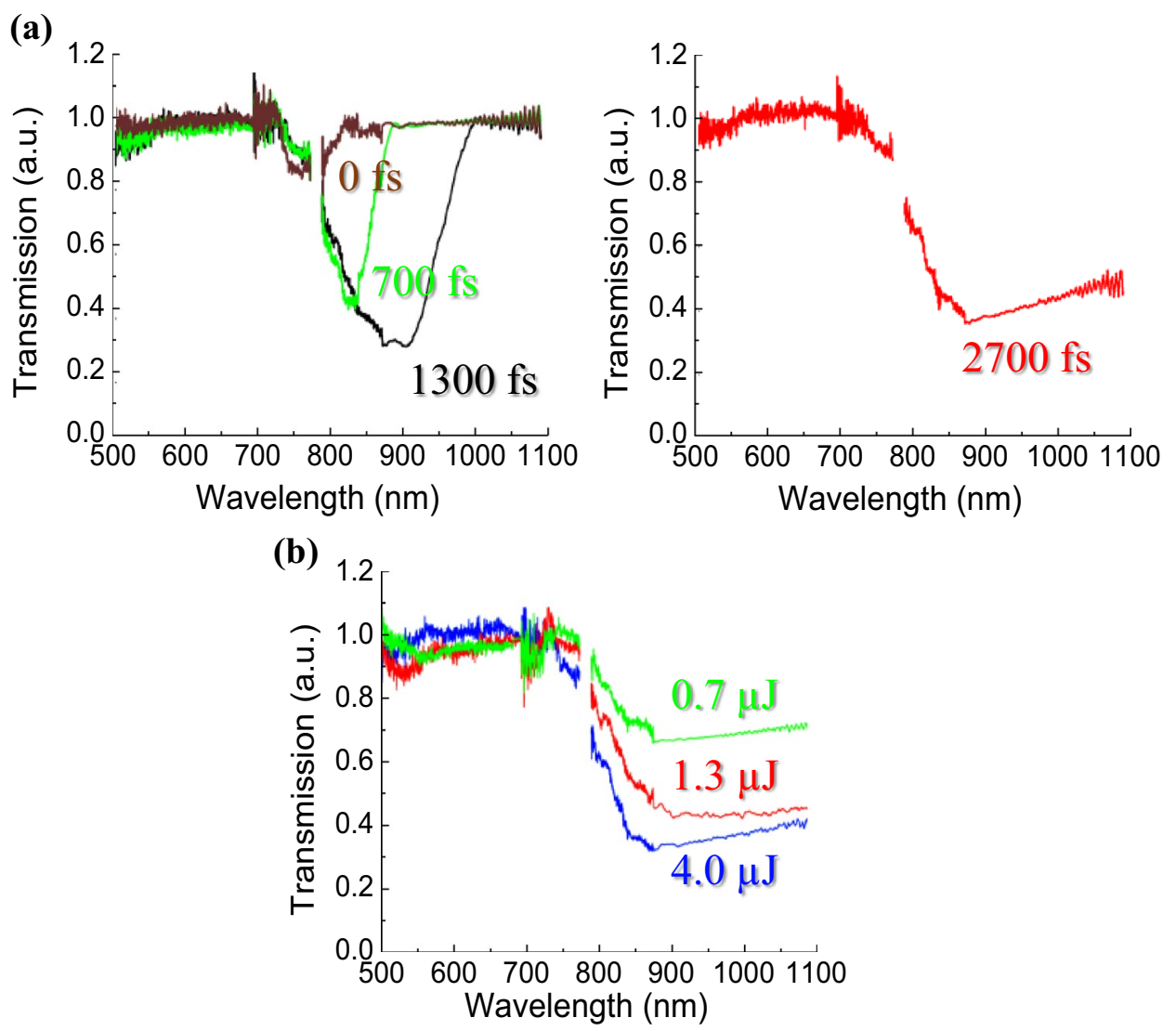

transmission decrease that indicates the onset of the pumpinduced plasma generation. We observe that the transient transmission gradually decreases for longer delays, i.e., 700 fs and $1300 \mathrm{fs}$. During the first 2 ps, we track the transmission at several wavelengths, all leading to the same rise time $\eta_{p} \approx 700$ fs. However, the minimum transmission is achieved earlier for shorter wavelengths, which indicates a time-mismatch effect as shown elsewhere [31]. This is due to the group velocity dispersion of the objective lens that induces a frequency-dependent time delay, i.e., a chirped probe. Moreover, the fact that the $\eta_{p}$ is longer than the pulse duration can be attributed to the combined effect of multiphoton ionization (MPI) and avalanche ionization (AI). In this way, seed electrons, which are excited via MPI, gain energy through inverse bremsstrahlung and further ionize more electrons via AI. This mechanism has been reported before in several dielectrics upon fs-laser excitation [33, 35, 36].

The shape of the spectrum achieved at 2.7 ps lasts up to 1 ns time delay. We attribute the step form to a pump-induced electron plasma that is transparent up to $725 \mathrm{~nm}$ and whose transmission steadily decreases to a value of 0.3 at $850 \mathrm{~nm}$. Figure $2 b$ shows the transmission of the excited material for three different pump energies, i.e., $0.7 \mu \mathrm{J}$ (green), 1.3 $\mu \mathrm{J}$ (red) and $4.0 \mu \mathrm{J}$ (blue). The curves present a lower transmission minima from $875 \mathrm{~nm}$ onwards as the pump energy decreases. We attribute the lower transmission to the higher electron density and larger plasma size achieved when using a higher pump energy. In contrast, the spectra display that the cutoff wavelength for the pump energy at $4.0 \mu \mathrm{J}$ is around $750 \mathrm{~nm}$, which is slightly larger for the curves collected using lower processing energies. Note here that $0.7 \mu \mathrm{J}$ is the only processing energy that leads to good-quality waveguides as shown in Fig. 1a. We will later use these curves to extract information of the plasma properties using the model explained below.

To accurately describe the system, we need to consider the total transmission of the SC probe beam through the plasma, $\mathcal{T}(\omega, \mathbf{r})$, which is the scalar product of the transmission coefficient $T(\omega, \mathbf{r})$ and the intensity distribution of the probe $\mathcal{M}(\omega, \mathbf{r})$,

$\mathcal{T}(\omega, \mathbf{r})=\mathcal{M}(\omega, \mathbf{r}) T(\omega, \mathbf{r})$,

The geometry on which we base our model consists of an electron plasma layer, parallel to the $z-y$ plane, and a SC probe that passes through it along the $x$-axis (see Fig.1). The Fresnel-Kirchhoff integral can be used to calculate the electric field of the focused pump beam at the $z-y$ plane from where one can retrieve a cross-section that yields a Gaussian curve [16]. Thus, it is reasonable to assume that the electron density will follow a Gaussian distribution. Note here that a cylindrical geometry could also be considered, 
thus using the Abel transformation. The profile extracted from the image of the laser-excited region shown in Fig. 1b further supports this assumption. We then approximate the electron plasma density distribution along the $y$-axis, $\rho_{e}(\mathbf{y})$, using a Gaussian distribution with a maximum electron density of $\rho_{\max }(y=0)$ that we use as a fit parameter. The maximum plasma thickness is calculated to be $d_{x}=0.7 \mu \mathrm{m}$ using the pump wavelength and the numerical aperture of the objective (NA = 0.4). We use Drude formula to compute the spectrally and spatially dependent dielectric function,

$\epsilon_{\mathrm{ex}}(\omega, \mathbf{y})=n_{g}(\omega)^{2}-\frac{\omega_{p}(\mathbf{y})^{2}}{\omega^{2}+i \omega / \tau}$

where $\omega$ is the frequency of the probe, $n_{g}(\omega)$ is the refractive index of fused silica, $\omega_{p}$ is the plasma frequency and $\tau$ is the scattering time that accounts both for $\tau_{e-e}$ and $\tau_{e-p}$. The refractive index of fused silica is calculated using the Sellmeier formula. The plasma frequency is calculated as $\omega_{p}(\mathbf{y})^{2}=\rho_{e}(\mathbf{y}) \mathrm{e}^{2} / m \epsilon_{o}$, where $m$ and $e$ are the reduced mass and the charge of the electron. The transmission of the plasma is calculated as $T(\omega, \mathbf{y})=(1-R(\omega, \mathbf{y}))^{2} \mathrm{e}^{-\alpha(\omega, \mathbf{y}) d_{x}}$, where $\alpha=2 \operatorname{Im}\left(n_{\mathrm{ex}}\right) \omega / c$ and $R$ is the reflectivity coefficient calculated using the Fresnel formula, which is squared due to the presence of two glass-plasma interfaces. Note here that in contrast to our previous model [31], we now consider a heterogeneous plasma by including the spatial distribution of the electron density.

It is crucial to also account for the frequency-dependent SC probe intensity distribution along the $y$-axis by introducing the following transmission function,

$\mathcal{M}(\omega, \mathbf{y}) \propto \frac{1}{w_{o}(\omega)^{2}} \mathrm{e}^{-\frac{2\left(y-y_{o}\right)^{2}}{w_{o}(\omega)^{2}}}$,

where the beam waist of the probe is a function of the laser wavelength $2 w_{o}=2 \lambda / \pi N A$, with NA $=0.55$. Details of the non-paraxial approximation in the context of laser materials processing can be found elsewhere [37]. We greatly attenuated the probe beam before being focused to minimize the non-linear response while still obtaining a good signal-tonoise ratio during detection. Thus, we only consider linear optics and discard a significant influence of non-linear effects associated with the probe beam propagation. We make sure there are no significant losses nor distortion of the beam by both measuring the input and output signal and imaging the probe's shape on a camera, see inset in Fig. $1 \mathrm{~b}$. Figure $3 \mathrm{a}$ shows the calculated beam waist of the probe as a function of the wavelength. The inset illustrates overlapped circles that picture the spatial probing mismatch for different wavelengths. In this way, a probe beam with a longer wavelength probes a bigger region of the laser-excited plasma, which presents a lower density especially near the edges (a)

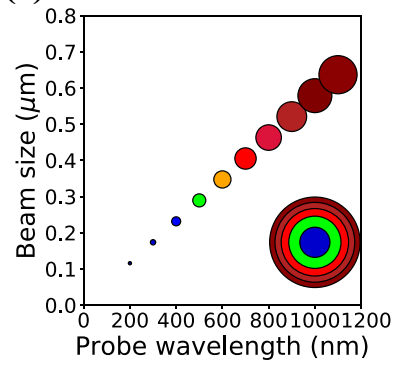

(c)

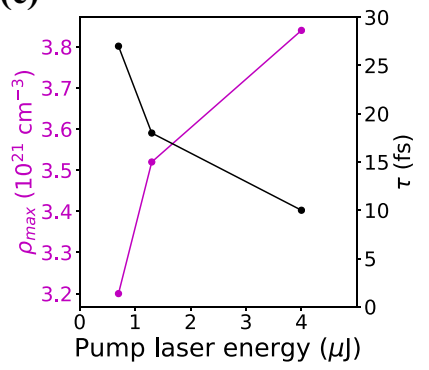

(b)

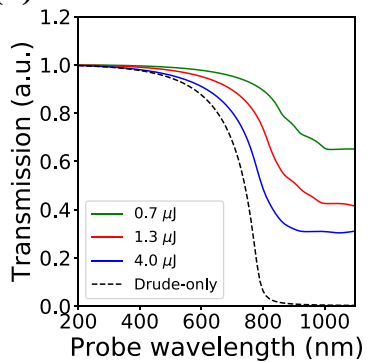

(d)

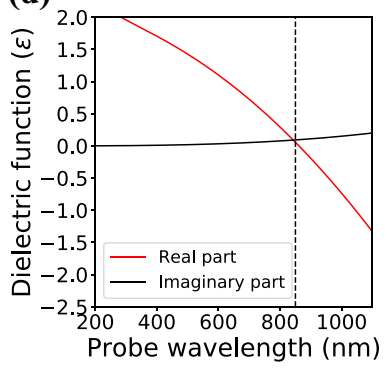

(e)

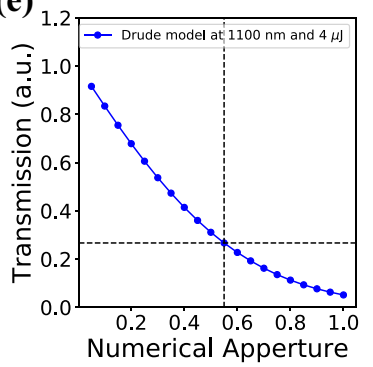

Fig. 3 a Probe beam size as a function of wavelength; the colored circles in the bottom-right corner illustrate the unequal probed area. b Transient transmission simulated using Drude-Sommerfeld model, with (solid lines) and without (black dashed line, using $4 \mu \mathrm{J}$ ) taking the probe beam size effects into account. The line color illustrates different pump energies as detailed in the legend. c Maximum electron density (left) and scattering time (right) used to compute the spectral curves in $\mathbf{b}$. d Computed dielectric function of the laser-induced electron plasma using a pump energy of $3.2 \mu \mathrm{J}$. e Transmission of the electron plasma at $1100 \mathrm{~nm}$ as a function of the NA of the microscope objective that focuses the SC probe beam

of the plasma distribution $\rho_{e}(\mathbf{y}>0.5 \mu \mathrm{m})$. A shorter probe wavelength is more tightly focused and probes mostly the center of the plasma distribution, where the local density is higher due to the influence of the laser peak power. We do not account for light scattering within the total extinction of the probe. This term depends on the precise shape and dielectric function of the illuminated laser-induced plasma and would certainly not be negligible when using higher laser pump energies as shown in reference [36].

Figure $3 \mathrm{~b}$ shows the calculated transmission spectra at different energies with (solid lines) and without (dashed line, using a pump energy of $4 \mu \mathrm{J}$ ) considering the SC probe beam size. The size-corrected curves are in agreement with the targeted experimental spectra presented in 
Fig. 2b. The computation using $\mathcal{M}(\omega, \mathbf{y})$ features a partially transparent plasma for wavelengths larger than 900 $\mathrm{nm}$. In contrast, we find an opaque plasma (dashed line) when considering an unrealistic perfect probe-plasma spatial overlap, i.e. $\mathcal{T}(\omega)=T(\omega)$.

From the fit, we obtain the electron density and the scattering time as a function of the processing energy as shown in Fig. 3c, whose convolution provides the energy balance in the solid. Our estimate of the density is slightly larger than our previously reported value for a $4 \mu \mathrm{J}$ excitation energy [31]. This is mainly due to the use of a more realistic electron density spatial distribution and plasma thickness. The reported scattering times range from 10 to $27 \mathrm{fs}$ and are slightly larger than other values reported in the literature that range from 1 to 23.3 fs $[3,38,39]$. We attribute this to the fact that our experiments offer a more restrictive target curve to fit, since we employ a broadband probe. In contrast, other works estimate the scattering rate and density of electrons combining measurements of the transient optical properties with Drude model using a monochromatic probe [28-30, 38, 40, 41], which leads to several acceptable combinations of $\rho$ and $\tau$.

We find that the processing energy of $0.7 \mu \mathrm{J}$, which leads to the fabrication of waveguides, generates a relatively lower density $\left(\rho=3.2 \times 10^{21} \mathrm{~cm}^{-3}\right)$ and a longer scattering time ( $\tau=27 \mathrm{fs}$ ), when compared to the results using higher energies. We also observe that higher processing energies naturally lead to a higher electron density, since the physical mechanisms that govern the excitation of free electrons scale both non-linearly and linearly with the laser intensity, via multiphoton and avalanche ionization, respectively. On the other hand, a shorter scattering time when using larger energy is indicative of a hotter electron plasma, according to Balling and Schou [24]. On the ps time scale, a hotter plasma with a high carrier density leads to a higher local temperature increase of the irradiated volume. Ultimately, this can produce the experimentally observed damage inside fused silica.

Figure $3 \mathrm{~d}$ illustrates the simulated spectral dependence of the complex dielectric function of the electron plasma ( $\rho=3.7 \times 10^{21} \mathrm{~cm}^{-3}$ and $\tau=12 \mathrm{fs}$ ). Logically, the real part of the dielectric function becomes negative, around $\approx 850 \mathrm{~nm}$, where we find the transition between a transparent and an opaque electron plasma as observed experimentally and predicted by the Drude theory [42]. Additionally, we show in Fig. 3e the effect of the NA of the objective on the transmission of the plasma at 1100 $\mathrm{nm}$. To minimize the effects of the chromatic aberration, we simply propose to use a microscope objective with a larger NA to focus the probe to a tighter spot. However, to perform this solution experimentally, one also needs to take into consideration the working distance of the objective as a limiting factor to probe laser-induced plasmas inside bulk dielectrics.

\section{Conclusions}

In conclusion, we have studied the temporally and spectrally resolved transmission of a fs-laser-induced electron plasma inside fused silica using several processing energies. We introduce a transmission function as the convolution of the material response and the spatial distribution of the probe considering the effect of chromatic aberration. We find a rise time of $700 \mathrm{fs}$ and fit a maximum electron density of $3.2 \times 10^{21} \mathrm{~cm}^{-3}$ and a scattering time of $27 \mathrm{fs}$ when using a processing energy that results in good-quality optical waveguides. Higher pump energies drive the formation of an electron plasma that qualitatively illustrate a similar step-like transmission and quantitatively present shorter scattering times and denser plasmas, which lead to the inscription of tracks of damaged material.

Acknowledgements We acknowledge fruitful discussions with Dries van Oosten, Luke Fletcher and Neil Troy. This work was supported by the National Science Foundation (NSF) Grants DMR-0801786 and DMR1206979.

Open Access This article is distributed under the terms of the Creative Commons Attribution 4.0 International License (http://creativeco mmons.org/licenses/by/4.0/), which permits unrestricted use, distribution, and reproduction in any medium, provided you give appropriate credit to the original author(s) and the source, provide a link to the Creative Commons license, and indicate if changes were made.

\section{References}

1. X. Liu, D. Du, G. Mourou, Laser ablation and micromachining with ultrashort laser pulses. IEEE J. Quant. Electron. 33(10), 1706-1716 (1997)

2. Y. Kondo, K. Nouchi, T. Mitsuyu, M. Watanabe, P.G. Kazansky, K. Hirao, Fabrication of long-period fiber gratings by focused irradiation of infrared femtosecond laser pulses. Opt. Lett. 24(10), 646-648 (1999)

3. L. Sudrie, A. Couairon, M. Franco, B. Lamouroux, B. Prade, S. Tzortzakis, A. Mysyrowicz, Femtosecond laser-induced damage and filamentary propagation in fused silica. Phys. Rev. Lett. 89, 186601 (2002)

4. R.R. Gattass, E. Mazur, Femtosecond laser micromachining in transparent materials. Nat. Photon. 2, 219 (2008)

5. C.-E. Athanasiou, Y. Bellouard, A monolithic micro-tensile tester for investigating silicon dioxide polymorph micromechanics, fabricated and operated using a femtosecond laser. Micromachines 6(9), 1365-1386 (2015)

6. C.-E. Athanasiou, M.-O. Hongler, Y. Bellouard, Unraveling brittle-fracture statistics from intermittent patterns formed during femtosecond laser exposure. Phys. Rev. Appl. 8, 054013 (2017) 
7. K.M. Davis, K. Miura, N. Sugimoto, K. Hirao, Writing waveguides in glass with a femtosecond laser. Opt. Lett. 21(21), 1729-1731 (1996)

8. K. Miura, J. Qiu, H. Inouye, T. Mitsuyu, K. Hirao, Photowritten optical waveguides in various glasses with ultrashort pulse laser. Appl. Phys. Lett. 71(23), 3329-3331 (1997)

9. K. Hirao, K. Miura, Writing waveguides and gratings in silica and related materials by a femtosecond laser. J. Non-crystal. Solids 239(1-3), 91-95 (1998)

10. V. Bharadwaj, A. Courvoisier, T.T. Fernandez, R. Ramponi, G. Galzerano, J. Nunn, M.J. Booth, R. Osellame, S.M. Eaton, P.S. Salter, Femtosecond laser inscription of bragg grating waveguides in bulk diamond. Opt. Lett. 42(17), 3451-3453 (2017)

11. E.N. Glezer, E. Mazur, Ultrafast-laser driven micro-explosions in transparent materials. Appl. Phys. Lett. 71(7), 882-884 (1997)

12. J.W. Chan, T.R. Huser, S.H. Risbud, D.M. Krol, Modification of the fused silica glass network associated with waveguide fabrication using femtosecond laser pulses. Appl. Phys. A 76(3), 367-372 (2003)

13. G.D. Marshall, M. Ams, M.J. Withford, Direct laser written waveguide-bragg gratings in bulk fused silica. Opt. Lett. 31(18), 2690-2691 (2006)

14. W.J. Reichman, D.M. Krol, L. Shah, F. Yoshino, A. Arai, S.M. Eaton, P.R. Herman, A spectroscopic comparison of femtosecondlaser-modified fused silica using kilohertz and megahertz laser systems. J. Appl. Phys. 99(12), 123112 (2006)

15. D.M. Krol, Femtosecond laser modification of glass. J. Non-Crystal. Solids 354(2), 416-424 (2008)

16. J. Hernandez-Rueda, J. Clarijs, D. van Oosten, D.M. Krol, The influence of femtosecond laser wavelength on waveguide fabrication inside fused silica. Appl. Phys. Lett. 110(16), 161109 (2017)

17. J. Hernandez-Rueda, J. Siegel, D. Puerto, M. Galvan-Sosa, W. Gawelda, J. Solis, Ad-hoc design of temporally shaped fs laser pulses based on plasma dynamics for deep ablation in fused silica. Appl. Phys. A 112(1), 185-189 (2013)

18. S.M. Eaton, H. Zhang, P.R. Herman, F. Yoshino, L. Shah, J. Bovatsek, A.Y. Arai, Heat accumulation effects in femtosecond laser-written waveguides with variable repetition rate. Opt. Express 13(12), 4708-4716 (2005)

19. T.T. Fernandez, G.D. Valle, R. Osellame, G. Jose, N. Chiodo, A. Jha, P. Laporta, Active waveguides written by femtosecond laser irradiation in an erbium-doped phospho-tellurite glass. Opt. Expresss 16(19), 15198 (2008)

20. T.T. Fernandez, S.M. Eaton, G.D. Valle, R.M. Vazquez, M. Irannejad, G. Jose, A. Jha, G. Cerullo, R. Osellame, P. Laporta, Femtosecond laser written optical waveguide amplifier in phosphotellurite glass. Opt. Express 18(19), 20289-20297 (2010)

21. L.B. Fletcher, J.J. Witcher, N. Troy, S.T. Reis, R.K. Brow, R.M. Vazquez, R. Osellame, D.M. Krol, Femtosecond laser writing of waveguides in zinc phosphate glasses. Opt. Mater. Express 1(5), 845-855 (2011)

22. J. Hernandez-Rueda, N.W. Troy, P. Freudenberger, R.K. Brow, D.M. Krol, Femtosecond laser-matter interactions in ternary zinc phosphate glasses. Opt. Mater. Express 8(12), 3622-3634 (2018)

23. B. Rethfeld, Unified model for the free-electron avalanche in laserirradiated dielectrics. Phys. Rev. Lett. 92, 187401 (2004)

24. P. Balling, J. Schou, Femtosecond-laser ablation dynamics of dielectrics: basics and applications for thin films. Rep. Progress Phys. 76(3), 036502 (2013)

25. S.W. Winkler, I.M. Burakov, R. Stoian, N.M. Bulgakova, A. Husakou, A. Mermillod-Blondin, A. Rosenfeld, D. Ashkenasi, I.V. Hertel, Transient response of dielectric materials exposed to ultrafast laser radiation. Appl. Phys. A 84(4), 413 (2006)
26. A. Mermillod-Blondin, J. Bonse, A. Rosenfeld, I.V. Hertel, Y.P. Meshcheryakov, N.M. Bulgakova, E. Audouard, R. Stoian, Dynamics of femtosecond laser induced voidlike structures in fused silica. Appl. Phys. Lett. 94(4), 041911 (2009)

27. A. Mermillod-Blondin, D. Ashkenasi, A. Lemke, M. Schwagmeier, A. Rosenfeld, Formation dynamics of ultra-short laser induced micro-dots in the bulk of transparent materials. Phys. Proced. 41, 776-780 (2013)

28. Q. Sun, H. Jiang, Y. Liu, W. Zhaoxin, H. Yang, Q. Gong, Measurement of the collision time of dense electronic plasma induced by a femtosecond laser in fused silica. Opt. Lett. 30(3), 320-322 (2005)

29. Q. Sun, H. Jiang, Y. Liu, W. Zhao-xin, H. Yang, Q. Gong, Diagnose parameters of plasma induced by femtosecond laser pulse in quartz and glasses. Front. Phys. China 1(1), 67-71 (2006)

30. Y. Hayasaki, M. Isaka, A. Takita, S. Juodkazis, Time-resolved interferometry of femtosecond-laser-induced processes under tight focusing and close-to-optical breakdown inside borosilicate glass. Opt. Express 19(7), 5725-5734 (2011)

31. J.J. Witcher, J. Hernandez-Rueda, D.M. Krol, Fs-laser processing of glass: plasma dynamics and spectroscopy. Int. J. Appl. Glass Sci. 6(3), 220-228 (2015)

32. K. Bergner, B. Seyfarth, K.A. Lammers, T. Ullsperger, S. Döring, M. Heinrich, M. Kumkar, D. Flamm, A. Tünnermann, S. Nolte, Spatio-temporal analysis of glass volume processing using ultrashort laser pulses. Appl. Opt. 57(16), 4618-4632 (2018)

33. J. Hernandez-Rueda, D. Puerto, J. Siegel, M. Galvan-Sosa, J. Solis, Plasma dynamics and structural modifications induced by femtosecond laser pulses in quartz. Appl. Surf. Sci. 258(23), 9389-9393 (2012). (EMRS 2011 Spring Symp J: Laser Materials Processing for Micro and Nano Applications)

34. J. Hernandez-Rueda, J. Siegel, M. Galvan-Sosa, A. Ruiz de la Cruz, M. Garcia-Lechuga, J. Solis, Controlling ablation mechanisms in sapphire by tuning the temporal shape of femtosecond laser pulses. J. Opt. Soc. Am. B 32(1), 150-156 (2015)

35. M.N. Christensen, J. Byskov-Nielsen, B.H. Christensen, P. Balling, Single-shot ablation of sapphire by ultrashort laser pulses. Appl. Phys. A 101(2), 279-282 (2010)

36. J. Hernandez-Rueda, D. van Oosten, Transient scattering effects and electron plasma dynamics during ultrafast laser ablation of water. Opt. Lett. 44(7), 1856-1859 (2019)

37. Q. Sun, H. Jiang, Y. Liu, Y. Zhou, H. Yang, Q. Gong, Effect of spherical aberration on the propagation of a tightly focused femtosecond laser pulse inside fused silica. J. Opti. A Pure Appl. Opt. 7(11), 655-659 (2005)

38. M.D. Feit, A.M. Komashko, A.M. Rubenchik, Ultra-short pulse laser interaction with transparent dielectrics. Appl. Phys. A 79(7), 1657-1661 (2004)

39. A.Q. Wu, I.H. Chowdhury, X. Xianfan, Femtosecond laser absorption in fused silica: numerical and experimental investigation. Phys. Rev. B 72, 085128 (2005)

40. S. Tzortzakis, L. Sudrie, M. Franco, B. Prade, A. Mysyrowicz, A. Couairon, L. Bergé, Self-guided propagation of ultrashort IR laser pulses in fused silica. Phys. Rev. Lett. 87(21), 213902 (2001)

41. X. Mao, S.S. Mao, R.E. Russo, Imaging femtosecond laserinduced electronic excitation in glass. Appl. Phys. Lett. 82(5), 697-699 (2003)

42. N.W. Ashcroft, N.D. Mermin, Solid state physics (2010)

Publisher's Note Springer Nature remains neutral with regard to jurisdictional claims in published maps and institutional affiliations. 